\title{
Doubled shot noise in disordered normal-metal-superconductor junctions
}

\author{
M J M de Jong \\ Phalips Research Laboratories, 5656 AA Endhoven, The Netherlands \\ and Instrtuut Lorentz, Unzversity of Leiden, 2300 RA Lerden, The Netherlands \\ C W J Beenakker \\ Instıtuut Lorentz, University of Lerden, 2300 RA Leiden, The Netherlands
}

(Received 28 February 1994)

\begin{abstract}
The low-frequency shot-noise power of a normal-metal-superconductor junction is studied for an arbitrary normal region Through a scattering approach, a formula is derived that expresses the shot-noise power in terms of the transmission eigenvalues of the normal region The noise power divided by the current is enhanced by a factor 2 with respect to its normal-state value, due to Cooper parr transport in the supeiconductor For a disordered normal region, it is still smaller than the Poisson norse as a consequence of nosseless open scattering channels
\end{abstract}

Electrical shot noise is the time dependent fluctuation of the current around the average $I$, due to the discreteness of the charge carriers The shot noise power $P$ gives information on the conduction process which is not contained in the resistance A well-known example is a vacuum diode, where $P=2 e|I| \equiv P_{\text {Poisson }}$ This tells us that the electrons traverse the diode in completely uncorrelated fashion, as in a Poisson process A noise power of $P_{\text {Poisson }}$ is the maximum value in the normal state $(\mathrm{N})$ In macroscopic samples shot noise is fully suppressed due to inelastic processes For sam ples of dimensions smaller than the inelastic scattering length shot noise is observable, but may be suppressed below $P_{\text {Pousson }}$ due to correlated electron transmission ${ }^{1}$ In this paper we investıgate theoretically the enhancement of shot noise at zero temperature in disordered normal-metal-superconductor (NS) junctions Navvely, one would expect $P=4 e|I|=2 P_{\text {Poisson }}$, since the current in the superconductor is carried by Cooper pairs in units of $2 e$ Instead, we find $P=\frac{2}{3} P_{\text {Poisson }}$, due to noiseless open scattering channels We also consider the more general case of a disordered region in series with a tunnel barrier In the absence of disorder we recover previous results by Khlus ${ }^{2}$ As far as we know, no measurements of shot noise in NS junctions have been reported yet Independent work on the problem has been carried out by Muzykantskı and Khmel'nitskı ${ }^{3}$ We furthermore would like to mention recent work on shot norse in a normalmetal-superconductor-normal-metal junction ${ }^{4}$

We first review the results for phase-coherent transport in the normal state The conductance at zero temperature and small applied voltage $V$ is given by the Landauer formula

$$
G_{\mathrm{N}}=G_{0} \operatorname{Tr} \mathbf{t} \mathrm{t}^{\dagger}=G_{0} \sum_{n=1}^{N} T_{n}
$$

where $G_{0} \equiv 2 e^{2} / h$ The matrix product $\mathrm{tt}^{\dagger}$ has eigenvalues $T_{n}, n=1,2, \quad, N$, with $N$ the number of scattering channels at the Fermi energy $E_{F}$ and $t$ the transmission matrix From current conservation it follows that
$T_{n} \in[0,1]$ A formula for the zero-frequency shot nolse power has been derived by Buttıker, ${ }^{5}$

$$
P_{\mathrm{N}}=P_{0} \operatorname{Tr}\left[\mathbf{t t}^{\dagger}\left(\mathbf{1}-\mathbf{t t}^{\dagger}\right)\right]=P_{0} \sum_{n=1}^{N} T_{n}\left(1-T_{n}\right)
$$

with $P_{0} \equiv 2 e|V| G_{0} \quad$ Equation (2) is the multichannel generalization of earlier single-channel formulas ${ }^{26}$ It is a consequence of the Paulı principle that closed $\left(T_{n}=0\right)$ as well as open $\left(T_{n}=1\right)$ scattering channels do not fluctuate and therefore give no contribution to the shot noise

In the case of a tunnel barrier, all transmission eigenvalues are small $\left(T_{n} \ll 1\right.$, for all $\left.n\right)$, so that the quadratıc terms in Eq (2) can be neglected Then it follows from comparison with $\mathrm{Eq}$ (1) that $P_{\mathrm{N}}=2 e|V| G_{\mathrm{N}}=$ $2 e|I|=P_{\text {Poisson }}$ In contrast, for a quantum point contact $P_{\mathrm{N}} \ll P_{\text {Poisson }} \quad$ Since on the plateaus of quantized conductance all the $T_{n}$ 's are either 0 or 1 , the shot noise is expected to be only observable at the steps between the plateaus ${ }^{6}$ This is indeed confirmed in an experiment by $\mathrm{Li}_{1}$ et $a l^{7}$ For a diffusive conductor of length $L$ much longer than the elastic mean free path $\ell$ it has been predicted that $P_{\mathrm{N}}=\frac{1}{3} P_{\text {Poisson }}$, as a consequence of noiseless open scatterıng channels ${ }^{8-11}$ Recently, an experimental observation of suppressed shot noise in a disordered wire has been reported 12

Now, let us turn to transport through a NS junction The conducting properties have originally been described by Blonder, Tinkham, and Klapwijk, ${ }^{13}$ and more recently in Refs 14-16 If the applied voltage is smaller than the superconducting gap $(e|V|<\Delta)$, the dissipative normal current is converted at the NS interface into dissipationless supercurrent, by means of Andreev reflection Electrons in the normal metal are retroreflected at the NS interface into holes, with the transfer of a Cooper pair to the superconducting condensate The scattering geometry is illustrated in the inset to $F_{1 g} 1$ Electrons and holes, incident from a reservoir via an ideal (impurityfree) lead, are scattered by an arbitrarily disordered, normal region in series with a superconductor The applied 


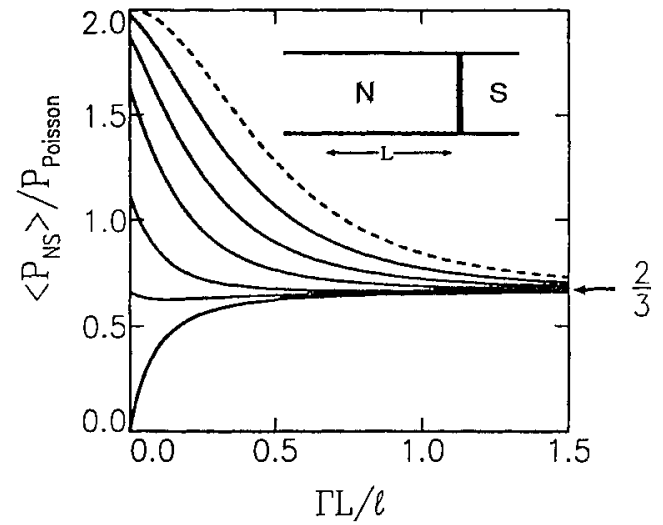

FIG. 1. The shot-noise power of a NS junction (in units of $P_{\text {Poisson }} \equiv 2 e|I|$ ) as a function of the length $L$ (in units of $\ell / \Gamma$ ), for barrier transparencies $\Gamma=1,0.9,0.8,0.6,0.4,0.2$ from bottom to top. The dashed curve gives the limiting result for $\Gamma \ll 1$. For $L=0$ the noise power varies as a function of $\Gamma$ according to Eq. (17), between doubled shot noise $\left(\left\langle P_{\mathrm{NS}}\right\rangle=4 e|I|\right)$ for high barriers $(\Gamma \ll 1)$ and zero in the absence of a barrier $(\Gamma=1)$. If $L$ increases the noise power approaches the limiting value $\left\langle P_{\mathrm{NS}}\right\rangle=\frac{4}{3} e|I|$ for each $\Gamma$. The inset shows schematically the NS junction.

voltage is taken to be small, and the temperature low, so that transmission of excitations into the superconductor is prohibited. All incident quasiparticles are therefore reflected back into the reservoir.

The calculation of the shot-noise power of the NS junction proceeds along the lines of Buttiker's method for normal-metal conductors. ${ }^{5}$ In the present case the scattering states are solutions of the Bogoliubov-de Gennes equation, ${ }^{13-16}$ rather than of a single-particle Schrödinger equation. The current operator in the lead towards the NS junction is given by

$$
\begin{aligned}
\hat{I}(t)= & \frac{e}{h} \sum_{\alpha, \beta} \int_{0}^{\infty} d \varepsilon \int_{0}^{\infty} d \varepsilon^{\prime} I_{\alpha \beta}\left(\varepsilon, \varepsilon^{\prime}\right) \\
& \times \hat{a}_{\alpha}^{\dagger}(\varepsilon) \hat{a}_{\beta}\left(\varepsilon^{\prime}\right) e^{i t\left(\varepsilon-\varepsilon^{\prime}\right) / \hbar},
\end{aligned}
$$

where $\hat{a}_{\alpha}^{\dagger}(\varepsilon)\left[\hat{a}_{\alpha}(\varepsilon)\right]$ is the creation (annihilation) operator of scattering state $\psi_{\alpha}(\varepsilon)$, and $I_{\alpha \beta}\left(\varepsilon, \varepsilon^{\prime}\right)$ is the matrix element of the current operator between states $\psi_{\alpha}(\varepsilon)$ and $\psi_{\beta}\left(\varepsilon^{\prime}\right)$. The quasiparticle energy $\varepsilon$ is measured with respect to $E_{F}$. In the lead, the state $\psi_{\alpha}$ consists of one incoming mode $\varphi_{\alpha}^{+}$and several, reflected, outgoing modes $\varphi_{\beta}^{-}$,

$$
\psi_{\alpha}(\varepsilon)=\varphi_{\alpha}^{+}(\varepsilon)+\sum_{\beta} r_{\beta \alpha}(\varepsilon) \varphi_{\beta}^{-}(\varepsilon) .
$$

The indices $\alpha, \beta$ denote mode number $(m)$ as well as whether it concerns electron $[\alpha=(m, e)]$ or hole $[\alpha=$ $(m, h)]$ propagation. The modes $\varphi^{+}, \varphi^{-}$are normalized to carry unit quasiparticle flux. The reflection amplitudes $r_{\beta \alpha}$ are contained in the unitary $2 N \times 2 N$ matrix $r$, which has the block form

$$
\mathbf{r}=\left(\begin{array}{ll}
\mathbf{r}_{e e} & \mathbf{r}_{e h} \\
\mathbf{r}_{h e} & \mathbf{r}_{h h}
\end{array}\right),
$$

where, e.g., the $N \times N$ submatrix $\mathbf{r}_{h e}$ contains the reflection amplitudes from incoming electrons to reflected holes. The unitarity of the reflection matrix corresponds to conservation of the number of quasiparticles. The conductance of the NS junction is given by ${ }^{15}$

$$
G_{\mathrm{NS}}=2 G_{0} \operatorname{Tr} \mathbf{r}_{h e} \mathbf{r}_{h e}^{\dagger} .
$$

In the zero-frequency limit we need the current-matrix elements $I_{\alpha \beta}(\varepsilon, \varepsilon)$ at equal energies. Following Ref. 5, we find

$$
I_{\alpha \beta}(\varepsilon, \varepsilon)=\left[\Lambda-\mathbf{r}^{\dagger}(\varepsilon) \Lambda \mathbf{r}(\varepsilon)\right]_{\alpha \beta} .
$$

The difference with Ref. 5 is the inclusion of the $2 N \times 2 N$ matrix $\Lambda$, defined by

$$
\Lambda \equiv\left(\begin{array}{cc}
-1 & 0 \\
0 & 1
\end{array}\right)
$$

which accounts for the opposite charges of electrons and holes. The average current $I$ can be determined from the expectation value of Eq. (3), using

$$
\left\langle\hat{a}_{\alpha}^{\dagger}(\varepsilon) \hat{a}_{\beta}\left(\varepsilon^{\prime}\right)\right\rangle=\delta_{\alpha \beta} \delta\left(\varepsilon-\varepsilon^{\prime}\right) f_{\alpha}(\varepsilon),
$$

with $f_{\alpha}(\varepsilon)$ the distribution function in the reservoir. At zero temperature and for $V<0$ one has for the electron $\left(f_{e}\right)$ and hole $\left(f_{h}\right)$ distribution functions

$$
f_{e}(\varepsilon)=\Theta(e|V|-\varepsilon), \quad f_{h}(\varepsilon)=0,
$$

with $\Theta(x)$ the unit-step function. The conductance $G_{\mathrm{NS}} \equiv \lim _{V \rightarrow 0} I / V$ can now easily be determined from Eqs. (3), (7), (9), and (10). This indeed provides the result Eq. (6) of Ref. 15, which serves as a check on the formalism.

We are now ready to compute the zero-frequency shotnoise power, defined by

$$
P_{\mathrm{NS}} \equiv 2 \int_{-\infty}^{\infty} d t\langle\Delta \hat{I}(t) \Delta \hat{I}(0)\rangle
$$

with $\Delta \hat{I}(t) \equiv \hat{I}(t)-I$. Substituting Eq. (3) and using Eq. (9) we find

$$
\begin{aligned}
P_{\mathrm{NS}}= & 2 \frac{e^{2}}{h} \int_{0}^{\infty} d \varepsilon \sum_{\alpha, \beta} I_{\alpha \beta}(\varepsilon, \varepsilon) I_{\beta \alpha}(\varepsilon, \varepsilon) \\
& \times f_{\alpha}(\varepsilon)\left[1-f_{\beta}(\varepsilon)\right] .
\end{aligned}
$$

Equation (12) can be evaluated through Eqs. (7) and (10). In the zero-temperature, zero-voltage limit we find, making use of the unitarity of $r$,

$$
\begin{aligned}
P_{\mathrm{NS}} & =4 P_{0} \operatorname{Tr}\left[\mathbf{r}_{h e} \mathbf{r}_{h e}^{\dagger}\left(1-\mathbf{r}_{h e} \mathbf{r}_{h e}^{\dagger}\right)\right] \\
& =4 P_{0} \sum_{n=1}^{N} \mathcal{R}_{n}\left(1-\mathcal{R}_{n}\right)
\end{aligned}
$$

where $\mathcal{R}_{n}$ is an eigenvalue of $\mathbf{r}_{h e} \mathbf{r}_{h e}^{\dagger}$, evaluated at $\varepsilon=0$.

It remains to relate the Andreev-reflection eigenvalues $\mathcal{R}_{n}$ to the scattering properties of the normal region. In the presence of time-reversal symmetry, i.e., in zero magnetic field, the eigenvalues $\mathcal{R}_{n}$ can be expressed entirely in terms of the transmission eigenvalues $T_{n}$ of the normal region: ${ }^{16}$ 


$$
\mathcal{R}_{n}=T_{n}^{2}\left(2-T_{n}\right)^{-2} \text {. }
$$

Equation (14) assumes a step function (at the NS interface) for the pair potential and neglects terms of order $\left(\triangle / E_{F}\right)^{2}$. Substitution into Eq. (6) yields the result of Ref. 16 for the conductance of the NS junction,

$$
G_{\mathrm{NS}}=G_{0} \sum_{n=1}^{N} \frac{2 T_{n}^{2}}{\left(2-T_{n}\right)^{2}}
$$

We now apply the same method to our result (13) for the shot-noise power, and find

$$
P_{\mathrm{NS}}=P_{0} \sum_{n=1}^{N} \frac{16 T_{n}^{2}\left(1-T_{n}\right)}{\left(2-T_{n}\right)^{4}}
$$

This is our main result. It is a general formula for arbitrary disorder potential in the normal region. As in the normal state, scattering channels which have $T_{n}=0$ or $T_{n}=1$ do not contribute to the shot noise. However, the way in which partially transmitting channels contribute is entirely different from the normal-state result (2). Before considering the case of a disordered conductor, we first briefly discuss the case of a planar tunneling barrier, which was previously studied by Khlus. ${ }^{2}$

A planar tunnel barrier is modeled by a channelindependent barrier transparency: $T_{n}=\Gamma$, for all $n$. It follows from Eq. (2), that for a normal conductor this would yicld $P_{\mathrm{N}}=(1-\Gamma) P_{\text {Porsson }}$, implying full Poisson noise for a high barrier $(\Gamma \ll 1)$. For the NS junction we find from Eqs. (15) and (16)

$$
P_{\mathrm{NS}}=P_{0} N \frac{16 \Gamma^{2}(1-\Gamma)}{(2-\Gamma)^{4}}=\frac{8(1-\Gamma)}{(2-\Gamma)^{2}} P_{\text {Poisson }}
$$

This agrees with the result of Khlus. ${ }^{2,17}$ If $\Gamma<2(\sqrt{2}-$ $1) \approx 0.83$, one observes a shot noise above the Poisson noise. For $\Gamma \ll 1$ one has

$$
P_{\mathrm{NS}}=4 e|I|=2 P_{\text {Poisson }},
$$

which is a doubling of the shot-noise power divided by the current with respect to the normal-state result. This can be interpreted as an uncorrelated current of $2 e$-charged particles.

We now turn to a NS junction with a disordered normal region, of length $L$ much greater than the mean free path $\ell$, but much smaller than the localization length, so that transport is in the metallic, diffusive regime. In Ref. 8 the average of the normal-state shot-noise power is computed. The method is applicable to any physical quantity of the form $\sum_{n} f\left(T_{n}\right)$ with $\lim _{T \rightarrow 0} f(T)=0$. (Such a quantity is called a linear statistic on the transmission eigenvalues.) Our formula (16) for the shot noise in the NS junction is of this form. According to Ref. 8 one has the general formula

$$
\left\langle\sum_{n=1}^{N} f\left(T_{n}\right)\right\rangle=\left\langle\sum_{n=1}^{N} T_{n}\right\rangle \int_{0}^{\infty} d x f\left(\cosh ^{-2} x\right) .
$$

Equation (19) is obtained from the relationship $T_{n}=$ $\cosh ^{-2}\left(L / \zeta_{n}\right)$ between the transmission eigenvalues and the channel-dependent localization lengths $\zeta_{n}$, and from the fact that $L / \zeta$ is uniformly distributed between 0 and $L / \ell \gg 1$. This uniform distribution is a general result of random-matrix theory, ${ }^{18}$ but has also been derived from a microscopic Green's function theory. ${ }^{11}$ The ensembleaveraged shot-noise power is now easily calculated by application of Eq. (19) to Eqs. (15) and (16), with the result

$$
\frac{\left\langle P_{\mathrm{NS}}\right\rangle}{\left\langle G_{\mathrm{NS}}\right\rangle}=\frac{2}{3} \frac{P_{0}}{G_{0}}
$$

hence

$$
\left\langle P_{\mathrm{NS}}\right\rangle=\frac{4}{3} e|I|=\frac{2}{3} P_{\text {Poisson }} .
$$

Equation (21) is twice the result in the normal state, but still smaller than the Poisson noise. Corrections to (21) are of lower order in $N$ and due to quantum-interference effects. ${ }^{10}$

Finally, we discuss a normal region which contains a disordered part as well as a tunnel barrier. This is most relevant to experiments, because in practice the NS interface is almost never ideal, but has a transparency $\Gamma<1$. However, the uniform distribution of $L / \zeta$ does not apply to such a system. In Refs. 11 and 19 the distribution of transmission eigenvalues of such a system is studied and an expression for $\left\langle G_{N S}\right\rangle$ as a function of $s \equiv L / \ell$ and $\Gamma$ is obtained. The shot-noise power can be derived in a similar fashion. Here we merely present the final expressions,

$$
\begin{aligned}
\left\langle G_{\mathrm{NS}}\right\rangle= & G_{0} N \frac{2 v^{\prime}(\phi)}{2 s v^{\prime}(\phi)-1} \\
\left\langle P_{\mathrm{NS}}\right\rangle= & P_{0} N\left(\frac{4 v^{\prime}(\phi)}{3\left[2 s v^{\prime}(\phi)-1\right]}-\frac{4 s v^{\prime \prime}(\phi)^{2}}{\left[2 s v^{\prime}(\phi)-1\right]^{5}}\right. \\
& \left.+\frac{2 v^{\prime \prime \prime}(\phi)}{3\left[2 s v^{\prime}(\phi)-1\right]^{4}}\right)
\end{aligned}
$$

with $v^{\prime}(\phi), v^{\prime \prime}(\phi), v^{\prime \prime \prime}(\phi)$ the first, second, and third derivative of

$$
v(\phi) \equiv \frac{\cos \phi}{2 / \Gamma+\sin \phi-1} .
$$

The auxiliary variable $\phi \in(0, \pi / 2)$ is the solution of

$$
\phi=2 s v(\phi) \text {. }
$$

The result is given in Fig. 1 , where $\left\langle P_{\mathrm{NS}}\right\rangle / P_{\text {Poisson }}$ is plotted against $\Gamma L / \ell$ for various $\Gamma$. Note the crossover from the ballistic (17) to the diffusive result (21). For a high barrier $(\Gamma \ll 1)$, the shot noise decreases from twice the Poisson noise to two-thirds the Poisson noise as the amount of disorder increases.

In summary, we have presented a theory for the shot noise in normal-superconductor junctions for arbitrary normal region. The general result (16) can be applied to many mesoscopic systems. We predict that for a disordered normal region the shot noise is suppressed below 
the Polsson noise by a factor $\frac{2}{3}$, due to the presence of noiseless open scattering channels This result is double the normal-state result, a consequence of the Cooper-parr transport in the superconductor For a normal region consisting of a disordered part and a barrier (at the NS interface), the shot-nosse power may vary between zero and a doubled Poisson noise, depending on the junction parameters We feel that observation of our predictions is within reach of present technology and presents a challenge for experımentalists

This research was supported by the Dutch Science Foundation NWO/FOM
${ }^{1}$ See, for example, $R$ Landauer and Th Martin, Physica B 175167 (1991), M Buttiker ibrd 175199 (1991), and references therein

${ }^{2} \mathrm{~V}$ A Khlus, Zh Eksp Teor Fiz 93, 2179 (1987) [Sov Phys JETP 66, 1243 (1987)]

${ }^{3} \mathrm{~B}$ A Muzykantskil and D E Khmel'nitskil (unpublished)

${ }^{4} \mathrm{U}$ Hanke, M Gisselfalt, Yu Galperm, M Jonson, R I Shekhter, and $\mathrm{K}$ A Chao (unpublished)

5 M Buttiker, Phys Rev Lett 65, 2901 (1990), Phys Rev B 46, 12485 (1992)

${ }^{6} \mathrm{G}$ B Lesovik, Pis'ma Zh Eksp Teor $\mathrm{F}_{1 \mathrm{z}}$ 49, 513 (1989) [JETP Lett 49, 592 (1989)]

7 Y P Ll, D C Tsui, J J Heremans, J A Simmons, and G W Weimann, Appl Phys Lett 57, 774 (1990)

${ }^{8} \mathrm{C} W \mathrm{~J}$ Beenakker and $\mathrm{M}$ Buttiker Phys Rev B 46, 1889 (1992)

${ }^{9} \mathrm{~K}$ E Nagaev, Phys Lett A 169, 103 (1992)

${ }^{10} \mathrm{M} J \mathrm{M}$ de Jong and $\mathrm{C}$ W J Beenakker, Phys Rev B 46, 13400 (1992)
11 Yu V Nazarov (unpublished)

${ }^{12} \mathrm{~F}$ Liefrink, J I Dijkhus, $M$ J $M$ de Jong $I \quad W$ Molenkamp and $H$ van Houten Phys Rev B 49, 14066 (1994)

${ }^{13} \mathrm{G}$ E Blonder, M Tinkham, and $\mathrm{T} M$ Klapwıj, Phys Rev B 25, 4515 (1982)

${ }^{14} \mathrm{C} J$ Lambert, J Phys Condens Matter 3, 6579 (1991)

${ }^{15} \mathrm{Y}$ Takane and $\mathrm{H}$ Ebisawa, J Phys Soc Jpn $\mathbf{6 1}, 2858$ (1992)

${ }^{16}$ C W J Beenakker, Phys Rev B 46, 12841 (1992)

${ }^{17}$ To see this, one must evaluate Eq (25a) of Ref 2 for $e V \ll$ $\Delta$ Note that the first exponent 2 should be -2

${ }^{18}$ For a review, see A D Stone, P A Mello, K A Muttalıb, and $J$ L Pichard, in Mesoscopıc Phenomena in Solvds, edited by $B$ L Al'tshuler, $P$ A Lee, and $R$ A Webb (North-Holland, Amsterdam, 1991)

${ }^{19} \mathrm{C}$ W J Beenakker, B Rejael, and J A Melsen, Phys Rev Lett 72, 2470 (1994) 